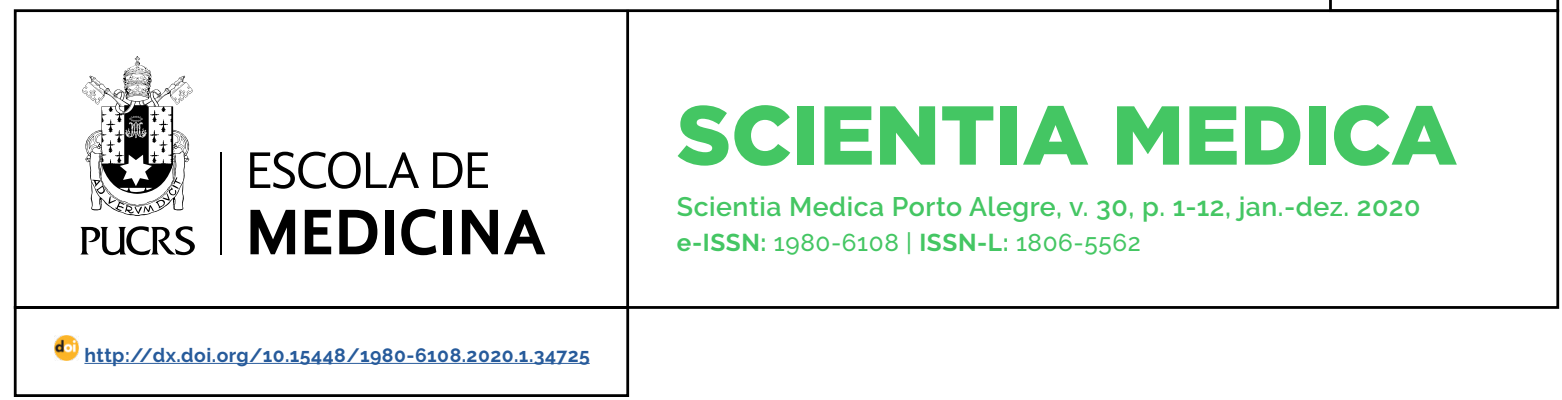

ARTIGO ORIGINAL

\title{
Impacto prognóstico das complicações ocorridas durante o transporte de crianças gravemente doentes
}

\author{
Prognostic impact of complications during transport of critically ill children
}

\section{Tabata Luna Garavazzo Tavares $^{1}$ \\ orcid.org/0000-0002-5271-9784}

lunagaravazzo@yahoo.com.br

\section{Alessandra Kimie Matsuno ${ }^{1}$ \\ orcid.org/0000-0002-3491-1641}

matsuno@fmrp.usp.br

\section{Fábio Carmona ${ }^{1}$}

orcid.org/0000-0001-5743-0325

carmona@fmrp.usp.br

\section{Ana Paula de Carvalho Panzeri Carlotti ${ }^{1}$} orcid.org/0000-0003-2760-8801 apcarlotti@fmrp.usp.br

\section{Carlos Henrique \\ Miranda ${ }^{1}$}

orcid.org/0000-0002-5968-4879

chmiranda@fmrp.usp.br

Recebido em: 5 jul. 2019 Aprovado em: 15 nov. 2019. Publicado em: 15 jul. 2020

\section{(c) (i)}

Artigo está licenciado sob forma de uma licença Creative Commons Atribuição 4.0 Internaciona.

\section{Resumo}

Objetivo: o transporte de crianças gravemente doentes envolve particularidades que aumentam o risco de complicações. O objetivo foi investigar o impacto de complicações registradas durante o transporte na mortalidade geral e na taxa de alta hospitalar.

Método: estudo realizado em duas etapas. A primeira foi um estudo transversal, no qual, através de entrevista padronizada, dirigida ao médico que admitiu essas crianças, foram identificadas potenciais complicações ocorridas durante o transporte. Três médicos independentes auditaram esses dados. A segunda etapa foi uma coorte prospectiva onde os pacientes divididos em dois grupos (com e sem complicações no transporte) foram seguidos prospectivamente.

Resultados: 143 crianças foram incluidas no estudo. Pelo menos uma complicação durante o transporte foi observada em 74 delas (52\%). A complicação mais prevalente foi relacionada a falhas no monitoramento e nos dispositivos (42\%). A ocorrência de complicações no transporte foi associada a maior mortalidade hospitalar (Hazard ratio - HR): 5,60; intervalo de confiança de 95\% (IC95\%): 1,26 26,65: $\mathrm{p}=0,013$ ) e a menor taxa de alta hospitalar (HR: 0,48; IC95\%: 0,31-0,74; $\mathrm{p}=$ $0,0007)$. Após regressão de Cox para ajuste de fatores de confusão, a presença de complicações permaneceu associada à mortalidade hospitalar (HR: 6,74: IC95\%: $1,40-32,34) ; p=0,017$ ), porém deixou de se associar com a taxa de alta hospitalar (HR: 0,76; IC95\%: 0,49 - 1,16; $p=0,213$ ).

Conclusão: presença de complicações durante o transporte pediátrico foi frequente na região metropolitana de Ribeirão Preto, São Paulo, Brasil. A ocorrência de complicações foi um preditor independente de mortalidade hospitalar.

Palaras-chave: transporte de pacientes, assistência pré-hospitalar, unidade de terapia intensiva pediátrica.

\section{Abstract}

Aims: Transport of critically ill children involves particularities that increase the risk of complications. The objective was to investigate the impact of these complications recorded during transport on overall mortality and hospital discharge rate.

Method: Two-step study: the first was a cross-sectional study, in which, through standardized interviews with the doctor who admitted these children, potential complications during transport were identified. Three independent doctors audited this data. The second step was a prospective cohort, where patients divided into two groups (with and without transport complications) were followed prospectively.

Results: One hundred and forty-three children were included in the study. At least one complication during transport was observed in 74 of them (52\%). The most frequent complication was related to monitoring and device failures (42\%). The occurrence of transport complications was associated with higher hospital mortality (Hazard ratio (HR): 5.60; 95\% confidence interval (95\% Cl: $1.26-26.65 ; \mathrm{p}=0.013$ ) and the lowest hospital discharge rate (HR: 0.48; 95\% Cl: $0.31-0.74 ; \mathrm{p}=0.0007$ ). After Cox regression to adjust for confounding factors, the presence of complications remained associated with hospital mortality (HR: 6.74; $95 \% \mathrm{Cl}: 1.40-32.34 ; \mathrm{p}=0.017$ ), but no remained associated with hospital discharge rate (HR: $0.76 ; 95 \% \mathrm{Cl}: 0.49-1.16 ; \mathrm{p}=0.213$ ). 
Conclusion: The presence of complications during pediatric transport was frequent in metropolitan region of Ribeirão Preto, São Paulo, Brazil. The occurrence of complications was an independent predictor for hospital mortality.

Keywords: patients' transportation, prehospital care, pediatric intensive care unit.

ABREVIATURAS: IC95\%, intervalo de confiança de 95\%; HR, Hazard ratio; PRISM2, Pediatric Risk of Mortality score 2; RR. risco relativo; SAMU, Serviço de Atendimento Móvel de Urgência.

\section{Introdução}

O aumento no número de transportes pré e inter-hospitalar de pacientes pediátricos gravemente doentes vem ocorrendo, provavelmente, secundário a maior sobrevida de crianças com doenças crônicas e à concentração de recursos tecnológicos em grandes centros hospitalares. Contudo, o transporte desses pacientes envolve várias particularidades que aumentam a possibilidade de complicações (1).

No Brasil, a maior parte desses transportes é realizado pelo Serviço de Atendimento Móvel de Urgência (SAMU), dentro do sistema público de saúde (2). Contudo, desconhece-se a prevalência de complicações durante esse tipo de transporte em nosso país, assim como, o impacto dessas complicações na mortalidade e na duração da internação hospitalar.

O objetivo do presente estudo foi determinar a prevalência e os tipos de complicações observadas durante o transporte pré e inter-hospitalar de crianças gravemente doentes, definir preditores para a ocorrência dessas complicações e avaliar o impacto dessas complicações em desfechos de mortalidade e de duração da hospitalização.

\section{Métodos}

Trata-se de um estudo observacional, dividido em duas etapas distintas. A primeira etapa correspondeu a um estudo transversal para avaliação da prevalência de complicações durante o transporte pré e inter-hospitalar de crianças gravemente doentes que foram identificadas na admissão hospitalar através de uma entrevista dirigida ao médico responsável pela admissão desses pacientes. A segunda etapa correspondeu a uma coorte prospectiva, na qual os pacientes identificados na primeira etapa foram divididos em dois grupos (presença ou ausência de complicações durante o transporte) e, prospectivamente, seguidos durante a internação até a ocorrência de óbito ou alta hospitalar.

Os participantes do estudo foram rastreados nas seguintes unidades do Hospital das Clínicas da Faculdade de Medicina de Ribeirão Preto da Universidade de São Paulo: Unidades de Terapia Intensiva Pediátrica da Unidade de Emergência e da Unidade Campus e Sala de Urgência Pediátrica da Unidade de Emergência. Os seguintes critérios de inclusão foram utilizados: pacientes de ambos os sexos com idade inferior a 15 anos; realização de transporte pré-hospitalar ou inter-hospitalar por meio de ambulância com a obrigatoriedade da presença de médico e diagnóstico médico de pelo menos uma condição clínica grave (insuficiência respiratória aguda ou crônica agudizada, choque circulatório, politraumatismo, traumatismo cranioencefálico, cardiopatias congênitas complexas, cetoacidose diabética). Foram excluidos os pacientes com os seguintes critérios: transporte realizado sem a presença de médico; crianças que procuraram diretamente $\mathrm{o}$ atendimento e que não foi realizado transporte antes da admissão hospitalar e transporte de paciente que já havia sido incluido no estudo previamente.

Para a identificação dos pacientes com esses critérios de inclusão, o pesquisador entrava em contato diariamente com as unidades participantes da pesquisa e questionava sobre a admissão de pacientes com os critérios de inclusão no estudo anotando a identificação do paciente e do médico responsável pela realização da admissão hospitalar. O pesquisador tinha um prazo de cinco dias para contatar o médico responsável pela admissão do paciente para a aplicação de um questionário dirigido para a identificação de complicações durante o transporte. O questionário somente era aplicado após a concordância do médico em participar do estudo e após a assinatura do Termo de Consentimento Livre e Esclarecido.

A presença de complicações durante o transporte foi buscada ativamente através de questionário 
padronizado pelos pesquisadores contendo os principais tipos de complicações esperadas durante o transporte de crianças gravemente doentes agrupadas de acordo com o sistema observado: complicações respiratórias, cardiovasculares, neurológicas, metabólicas, relacionadas ao trauma e a dispositivos. Durante a aplicação desse questionário, caso fosse identificada pelo médico responsável pela admissão alguma intercorrência durante o transporte, essa ocorrência era detalhada de forma descritiva.

Esse questionário fez a busca ativa das seguintes complicações dividas pelo respectivo sistema relacionado:

1. complicações respiratórias: necessidade de ventilação mecânica com ausência de ventilador, presença de secreção excessiva, deslocamento da cânula traqueal para o esôfago, cânula traqueal seletiva, tamanho da cânula inadequado, ausência de intubação na presença de insuficiência respiratória franca, interrupção do fornecimento de oxigênio, fornecimento excessivo de oxigênio em casos específicos de cardiopatias congênitas e sedação inadequada em pacientes intubados;

2. complicações cardiovasculares: choque não identificado definido pela pressão arterial abaixo do percentil 90 e tempo de enchimento capilar maior que dois segundos ou pulso periférico fino com extremidades frias ou pulso amplo e perfusão rápida; choque não tratado adequadamente definido pela expansão volêmica inferior a $60 \mathrm{~mL} / \mathrm{kg}$ em uma hora nos casos em que houvesse indicação; uso inadequado de drogas vasoativas definido por subdoses ou overdoses; choque séptico sem administração de antibioticoterapia na primeira hora; assistência à parada cardiorrespiratória em inconformidade com as diretrizes propostas pelo Pediatric Advanced Life Support;

3. complicações neurológicas: rebaixamento do nível de consciência com escala de coma de Glasgow $\leq 8$ sem pro- teção da via aérea, crise convulsiva não tratada ou com tratamento inadequado;

4. complicações metabólicas: hipotermia definida pela temperatura axilar menor que $36^{\circ} \mathrm{C}$; hipertermia definida pela temperatura axilar maior que $37,8^{\circ} \mathrm{C}$; hipoglicemia definida como glicemia ou glicosimetria capilar menor que $40 \mathrm{mg} /$ $\mathrm{dl}$ em neonatos e menor que $60 \mathrm{mg} / \mathrm{dl}$ em lactentes e crianças; hiperglicemia definida pela glicemia ou glicosimetria capilar maior que $150 \mathrm{mg} / \mathrm{dl}$;

5. complicações relacionadas ao trauma: pacientes com trauma cranioencefálico ou politraumatizados sem colar cervical, ausência de prancha rigida nos casos de politraumatizados e presença de fraturas não imobilizadas;

6. complicações relacionadas a dispositivos e monitorização: ausência de monitorização da pressão arterial, ausência de oximetria de pulso, ausência de monitor cardíaco, ausência ou perda de acesso venoso em pacientes com necessidade de administração de fluidos ou drogas endovenosas.

Os dados clínicos, demográficos e laboratoriais dos pacientes foram obtidos a partir do prontuário médico. Todos esses casos foram auditados por três médicos independentes, que após a descrição dos fatos observados durante o transporte das crianças julgaram se havia ocorrido ou não alguma complicação. Nos casos onde não houve unanimidade de opiniões, prevaleceuse a decisão da maioria. Somente após essa auditoria interna, os pacientes foram divididos em dois grupos: com presença e ausência de complicações durante o transporte hospitalar.

Para determinação da gravidade da condição clínica do paciente utilizou-se do escore Pediatric Risk of Mortality 2 (PRISM2), um escore padrão de gravidade desenvolvido para crianças gravemente doentes, composto por 14 variáveis fisiológicas medidas rotineiramente em pediatria, e calculado utilizando-se o pior valor durante as primeiras 24 horas da admissão. Para o cálculo da distância aproximada entre a unidade de origem e a unidade receptora utilizou-se o aplicativo 
Google Maps. O trabalho foi aprovado pelo Comitê de Ética em pesquisa do hospital de Clínicas da Faculdade de Medicina de Ribeirão Preto da Universidade de São Paulo, ofício n. ${ }^{\circ}$ 2694/2014 e seguiu os preceitos da declaração de Helsinki.

O método de Freedman foi utilizado para estimativa do tamanho amostral. Considerando um nivel de significância de $5 \%$, um poder de 80\% e que a presença de complicações durante o transporte duplicaria a taxa de mortalidade (hazard ratio (HR) de 2), estimou ser necessária a inclusão de 72 pacientes neste estudo.

As variáveis categóricas foram expressas em porcentagem. As variáveis quantitativas com distribuição normal foram expressas em média e em desvio padrão, e aquelas com outro tipo de distribuição foram expressas em mediana e em intervalo interquartil. Os testes de Shapiro-Wilk e de D Agostino-Pearson foram utilizados para avaliar se as variáveis apresentavam distribuição normal. Para a comparação de duas variáveis contínuas com distribuição normal utilizouse o teste $\mathrm{t}$ de Student não pareado e para a comparação de duas variáveis contínuas sem distribuição normal utilizou-se o teste de MannWhitney. Para a comparação das variáveis categóricas aplicou-se o teste exato de Fisher nas tabelas 2x2 ou teste do qui-quadrado nas tabelas maiores que 2x2. Foram estimados os riscos relativos (RR) brutos (análise univariada) e ajustados (modelos múltiplos) para as seguintes variáveis: peso menor que $10 \mathrm{~kg}$, distância maior que $100 \mathrm{~km}$, doença respiratória e presença de comorbidades através de modelo de regressão log-binomial com o auxilio do software SAS 9.3. Para avaliação do impacto das complicações durante o transporte na taxa de mortalidade e de alta hospitalar na coorte prospectiva foram construidas as curvas de Kaplan-Meier, calculado o hazard ratio e aplicado o teste de logrank. Na análise de sobrevida, para correção de potenciais fatores de confusão, foram construidas as curvas de Kaplan-Meier, ajustadas para as seguintes variáveis: peso menor que $10 \mathrm{~kg}$, distância do transporte, doença respiratória e presença de comorbidades utilizando-se do modelo de regressão proporcional de Cox. Considerou-se um p valor <0,05 como estatisticamente significativo em toda a análise. O software estatístico Stata versão 13.1 foi utilizado para a análise dos dados e a construção dos gráficos.

\section{Resultados}

O transporte de 143 crianças gravemente doentes foi avaliado no periodo de julho de 2014 a julho de 2015. As características demográficas e clínicas desses pacientes são mostradas na Tabela 1.

Em relação às características demográficas, uma maior porcentagem de pacientes com peso entre $3-10 \mathrm{~kg}$ foi observada no grupo com complicações (42\% versus $23 \% ; p=0,01$ ). Não foi observada diferença entre outras faixas de peso, sexo e faixa etária. Quanto às características do transporte, verificou-se maior distância do transporte no grupo com complicações comparado com o grupo sem complicações (36 km versus $23 \mathrm{Km} ; \mathrm{p}=0,02$ ). Não houve diferença estatística em relação ao tipo de equipe que realizou o transporte e a ocorrência de complicações. Analisando as caracteristicas clínicas, uma maior prevalência de doenças respiratórias foi observada no grupo com complicações durante o transporte (47\% versus 29\%; $p=0,02$ ), assim como, uma maior prevalência de comorbidades nesse grupo (53\% versus $26 \%$; $p=0,001$ ) (Tabela 1). 
TABELA 1 - Caracteristicas demográficas, clínicas e relacionadas ao transporte dos pacientes incluídos no estudo, com e sem complicações registradas durante o transporte

\begin{tabular}{|c|c|c|c|c|}
\hline \multirow[t]{2}{*}{ Variável } & Total & Sem complicação & $\begin{array}{c}\text { Com } \\
\text { complicação }\end{array}$ & \multirow{2}{*}{ Valor de $p$} \\
\hline & $n=143$ & $n=69$ & $n=74$ & \\
\hline Sexo masculino; $\mathrm{n}(\%)$ & $81(57)$ & $38(55)$ & $43(58)$ & 0,71 \\
\hline Faixa etária, n(\%) & & & & 0,41 \\
\hline$<1$ mês & $6(04)$ & $3(04)$ & $3(04)$ & \\
\hline 1 mês - 2 anos & $52(37)$ & $26(38)$ & $26(35)$ & \\
\hline 2 anos -7 anos & $39(27)$ & $19(28)$ & $20(27)$ & \\
\hline$\geq 7$ anos & $46(32)$ & $21(30)$ & $25(34)$ & \\
\hline Peso (em Kg), n(\%) & & & & 0,01 \\
\hline$<3$ & $7(05)$ & 2(03) & $5(07)$ & 0,28 \\
\hline $3-10$ & $47(33)$ & 16(23) & $31(42)$ & 0,01 \\
\hline $10-20$ & $46(32)$ & $26(38)$ & $20(27)$ & 0,17 \\
\hline$\geq 20$ & $43(30)$ & $25(36)$ & 18(24) & 0,12 \\
\hline \multicolumn{5}{|l|}{ Transporte } \\
\hline Municipal, n(\%) & $60(42)$ & $33(48)$ & $27(37)$ & 0,17 \\
\hline Pré-hospitalar, n(\%) & $108(76)$ & $57(40)$ & $51(36)$ & 0,08 \\
\hline Inter-hospitalar, n(\%) & $35(24)$ & 12(08) & $23(16)$ & 0,08 \\
\hline Distância (em Km)* & $28(6-81)$ & $23(4-58)$ & $36(6-104)$ & 0,02 \\
\hline \multicolumn{5}{|l|}{ Equipe responsável } \\
\hline SAMU, n(\%) & $66(46)$ & $32(46)$ & $34(46)$ & 0,95 \\
\hline Nossa instituição, n(\%) & 12(08) & $4(06)$ & $8(11)$ & 0,30 \\
\hline Outros, $\mathrm{n}(\%)$ & $65(45)$ & $33(46)$ & $32(43)$ & 0,58 \\
\hline \multicolumn{5}{|l|}{ Diagnóstico clínico, n(\%) } \\
\hline Doença infecciosa & $71(50)$ & $29(42)$ & $42(57)$ & 0,08 \\
\hline Doença respiratória & $55(38)$ & 20(29) & $35(47)$ & 0,02 \\
\hline Doença neurológica & $41(29)$ & $24(35)$ & $17(23)$ & 0,12 \\
\hline Doença cardiovascular & 25(17) & $11(16)$ & 14(19) & 0,64 \\
\hline Doença gastrointestinal & $19(13)$ & 12(17) & $7(10)$ & 0,16 \\
\hline Trauma & $11(08)$ & $4(06)$ & $7(10)$ & 0,41 \\
\hline Outros & $23(16)$ & $8(12)$ & $15(20)$ & 0,16 \\
\hline Comorbidades, $n(\%)$ & $57(40)$ & $18(26)$ & $39(53)$ & 0,001 \\
\hline
\end{tabular}

"mediana e intervalo interquartil.

SAMU, Serviço de atendimento móvel de urgência. 
A Tabela 2 mostra a distribuição detalhada de todas as 450 complicações registradas durante o transporte. A presença de pelo menos uma complicação durante o transporte foi observada em 74 pacientes (52\%). No total, 450 complicações foram registradas, pois 23 pacientes (16\%) apresentaram duas complicações e 34 pacientes (24\%) apresentaram três ou mais complicações. Em relação ao tipo de complicação, verificouse a seguinte distribuição: complicações relacionadas a dispositivos e à monitorização (42\%); complicações cardiovasculares (20\%); complicações respiratórias (18\%); complicações metabólicas (10\%); complicações relacionadas ao trauma (4\%) e complicações neurológicas (4\%).

TABELA 2 - Distribuição de todas as complicações registradas durante o transporte das crianças gravemente doentes de acordo com a classificação proposta pelos autores

\begin{tabular}{|c|c|}
\hline Variável & $\begin{array}{c}\text { Total } \\
n=450\end{array}$ \\
\hline Respiratória, n(\%) & $83(18)$ \\
\hline Secreção em via aérea & $23(5)$ \\
\hline Sedação insuficiente & $12(3)$ \\
\hline Cânula traqueal de tamanho inadequado & 12(3) \\
\hline Intubação indicada e não realizada & $12(3)$ \\
\hline Intubação dificil & $8(2)$ \\
\hline Intubação traqueal seletiva & $7(2)$ \\
\hline $\begin{array}{l}\text { Interrupção de fornecimento de } \\
\text { oxigênio }\end{array}$ & $5(1)$ \\
\hline Intubação esofágica & $2(0,5)$ \\
\hline Oxigênio em excesso & $2(0,5)$ \\
\hline Metabólica, n(\%) & $47(10)$ \\
\hline Hipertermia & 18(4) \\
\hline Hipotermia & $11(2)$ \\
\hline Hiperglicemia & $10(2)$ \\
\hline Hipoglicemia & $8(2)$ \\
\hline Cardiovascular, $\mathrm{n}(\%)$ & $92(20)$ \\
\hline Infusão de volume insuficiente & $25(5)$ \\
\hline Choque não tratado & $23(5)$ \\
\hline Choque não identificado & 18(4) \\
\hline
\end{tabular}

\begin{tabular}{|c|c|}
\hline Variável & $\begin{array}{l}\text { Total } \\
n=450\end{array}$ \\
\hline Drogas vasoativas inadequadas & $6(1)$ \\
\hline Sepse sem antibióticos & $4(1)$ \\
\hline $\begin{array}{l}\text { Ressuscitação cardiopulmonar inade- } \\
\text { quada }\end{array}$ & $4(1)$ \\
\hline Arritmia não tratada & $2(0,5)$ \\
\hline $\begin{array}{l}\text { Parada cardiorrespiratória não identi- } \\
\text { ficada }\end{array}$ & $1(0,5)$ \\
\hline Outras & $9(2)$ \\
\hline Monitorização e dispositivos, n(\%) & 191(42) \\
\hline $\begin{array}{l}\text { Ausência de monitor de pressão } \\
\text { arterial }\end{array}$ & $74(16)$ \\
\hline Ausência de monitor cardiaco & 73(16) \\
\hline Ausência de acesso venoso & $21(5)$ \\
\hline Ausência de oximetria de pulso & 15(3) \\
\hline Outras & $8(2)$ \\
\hline Neurológica, n(\%) & $19(4)$ \\
\hline $\begin{array}{l}\text { Escala de Glasgow } \leq 8 \text { sem proteção } \\
\text { de via aérea }\end{array}$ & $4(1)$ \\
\hline Crise convulsiva não tratada & $5(1)$ \\
\hline $\begin{array}{l}\text { Tratamento inadequado de crise } \\
\text { convulsiva }\end{array}$ & $6(1)$ \\
\hline $\begin{array}{l}\text { Medidas iniciais traumatismo cranioe- } \\
\text { ncefálico não realizadas }\end{array}$ & $4(1)$ \\
\hline Trauma, $n(\%)$ & $18(4)$ \\
\hline Ausência de prancha rígida & $7(2)$ \\
\hline Ausência de colar cervical & $8(2)$ \\
\hline Ausência de imobilização de fraturas & 3(1) \\
\hline
\end{tabular}

O número total de complicações foi superior ao número de pacientes incluidos porque muitos pacientes apresentaram mais de uma complicação registrada durante o transporte.

Em relação à primeira etapa deste estudo, comparando-se o grupo com complicações versus o grupo sem complicações, na análise univariada foram identificados os seguintes preditores de risco para ocorrência de complicações durante o transporte: peso <10Kg (RR: 1,52; intervalo de confiança de 95\% (IC95\%): 1,11 - 2,09); distância >100Km (RR: 1,67; IC95\%: 1,16 - 2,40); presença de doença respiratória (RR: 1,46; 
IC95\%: 1,06 - 1,95) e presença de comorbidades (RR: 1,68; IC95\%: 1,23 - 2,30). Após análise multivariada utilizando-se de modelo de regressão log-binomial, em que todas essas variáveis foram incluidas, nenhuma delas se manteve como preditor independente para ocorrência de complicações durante o transporte (Tabela 3).

TABELA 3 - Análise de potenciais preditores de risco para ocorrência de complicação durante o transporte na análise univariada e na análise multivariada ajustada para peso $<10 \mathrm{Kg}$, distância $>100 \mathrm{Km}$, doenças respiratórias e presença de comorbidades

\begin{tabular}{|c|c|c|c|c|c|c|}
\hline & \multicolumn{3}{|c|}{ Univariada } & \multicolumn{3}{|c|}{ Multivariada } \\
\hline Variáveis & $\mathrm{RR}$ & IC95\% & $\mathrm{p}$ & $\mathrm{RR}$ & IC95\% & $\mathrm{p}$ \\
\hline \multicolumn{7}{|l|}{ Peso (Kg) } \\
\hline$>10^{*}$ & $1,00^{*}$ & & & & & \\
\hline$<10$ & 1,52 & $1,11-2,90$ & 0,009 & 1,00 & $0,83-1,22$ & $>0,05$ \\
\hline \multicolumn{7}{|l|}{ Distância (Km) } \\
\hline$<15^{*}$ & $1,00^{*}$ & & & & & \\
\hline $16-50$ & 1,14 & $0,74-1,77$ & $>0,05$ & 1,09 & $0,92-1,30$ & $>0,05$ \\
\hline $51-100$ & 1,06 & $0,64-1,77$ & $>0,05$ & 1,07 & $0,85-1,36$ & $>0,05$ \\
\hline$>100$ & 1,67 & $1,16-2,40$ & 0,010 & 1,10 & $0,86-1,39$ & $>0,05$ \\
\hline \multicolumn{7}{|l|}{ Equipe transporte } \\
\hline Nossa instituição & 1,35 & $0,84-2,17$ & $>0,05$ & 1,07 & $0,71-1,62$ & $>0,05$ \\
\hline SAMU & 1,05 & $0,74-1,47$ & $>0,05$ & 1,05 & $0,89-1,23$ & $>0,05$ \\
\hline \multicolumn{7}{|l|}{ Diagnósticos } \\
\hline Doenças respiratórias & 1,43 & $1,06-1,95$ & 0,020 & 1,00 & $0,81-1,24$ & $>0,05$ \\
\hline Comorbidades & 1,68 & $1,23-2,30$ & 0,001 & 1,05 & $0,86-1,28$ & $>0,05$ \\
\hline
\end{tabular}

* Referência

RR, risco relativo, IC, intervalo de confiança, SAMU, Serviço de Atendimento Móvel de Urgência

A presença de complicação durante o transporte foi associada com: maior taxa de mortalidade hospitalar (18\% versus 03\%; $p=0,005$ ); maior duração da internação hospitalar total (7,5 dias versus 05 dias; $p=0,02$ ); e maior duração da internação na unidade de terapia intensiva (04 dias versus oo dias; $\mathrm{p}=0,02)$. Também foi observada uma maior gravidade dos casos na admissão hospitalar no grupo com complicação em relação ao grupo sem complicação avaliado através do escore PRISM2 (8,5 versus 4,0; $p=0,0001$ ). No grupo com complicação, 41\% dos pacientes apresentaram escore PRISM2 maior que 10 comparado com $17 \%$ no grupo sem complicação; $p=0,002$.
Na análise de sobrevida através da curva de Kaplan-Meier, a ocorrência de complicação foi estatisticamente associada com o aumento da mortalidade intra-hospitalar IHR: 5,60; IC95\%: 1,26 - 26,65; $p=0,013$ ) (Figura 1A). Após ajuste para potenciais fatores de confusão, utilizando-se do modelo de regressão proporcional de Cox, observou-se que a ocorrência de complicação durante o transporte se manteve como preditor independentemente de mortalidade (HR: 6.74; IC95\%: 1,40 - 32,34; $p=0,017$ ) (Figura 1B). 

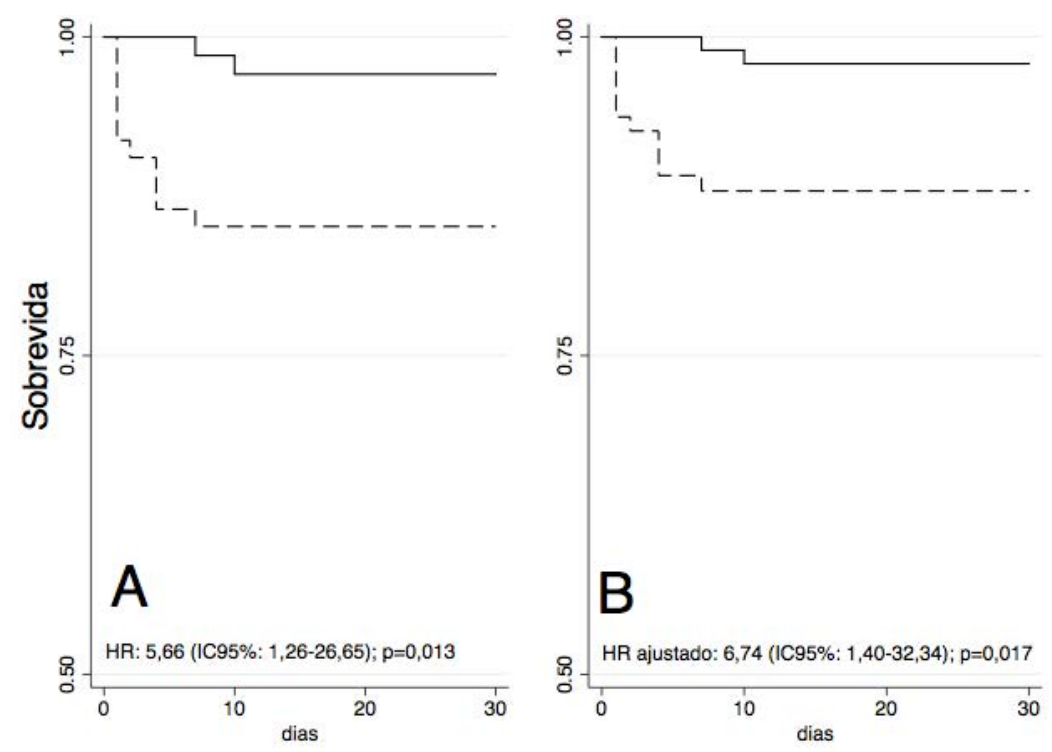

Figura 1 - Curvas de Kaplan-Meier comparando a sobrevida ao longo de 30 dias no grupo de pacientes sem complicação durante o transporte (linha continua) versus o grupo de pacientes com complicação durante o transporte (linha tracejada). Curva A não foi ajustada e a curva B foi ajustada através de regressão de Cox para peso < $10 \mathrm{Kg}$, distância >100 Km, doença respiratória e comorbidades. (HR, Hazzard Ratio; IC95\%, intervalo de confiança de 95\%)

Em relação ao tempo para a alta hospitalar, analisado através das curvas de Kaplan-Meier, inicialmente, verificou-se maior tempo para a alta hospitalar no grupo com complicações (HR: 0,48; IC95\%: 0,31 - 0,74: $p=0,0007$ ) (Figura 2A). Contudo, após ajuste para os mesmos fatores de confusão através do modelo de regressão proporcional de Cox. o impacto da complicação durante o transporte no tempo para a alta hospitalar não foi mais observado (HR: 0,76; IC95\%: 0,49 - 1,16; p=0,213). (Figura 2B).
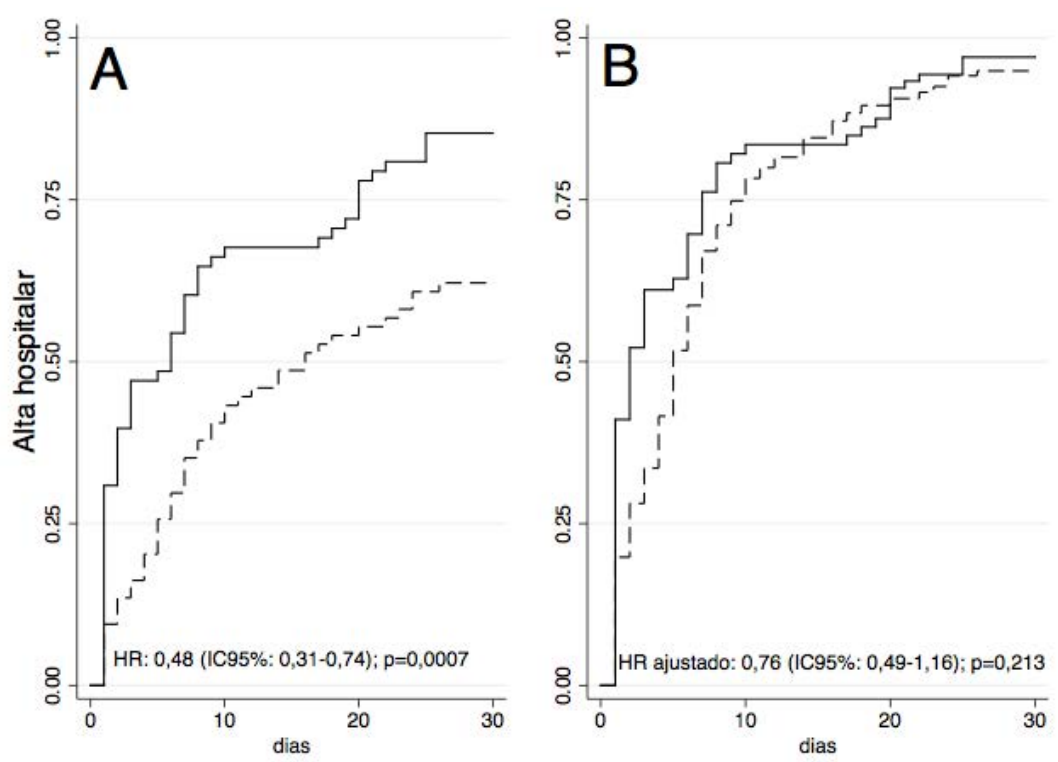

Figura 2 - Curvas de Kaplan-Meier comparando o tempo até a alta hospitalar no grupo de pacientes sem complicação durante o transporte (linha contínua) versus o grupo de pacientes com complicação durante o transporte (linha tracejada). Curva A não foi ajustada e a curva B foi ajustada através de regressão de Cox para peso < 10 Kg, distância > 100 Km, doença respiratória e comorbidades. (HR, Hazzard Ratio; IC95\%, intervalo de confiança de 95\%) 


\section{Discussão}

Esta investigação avaliou o transporte pré e inter-hospitalar de 143 crianças gravemente doentes e mostrou a presença de pelo menos uma complicação durante o transporte em 74 delas (52\%). As complicações mais frequentes foram relacionadas à falha de dispositivos e à monitorização (42\%). Na análise univariada, os seguintes preditores para a ocorrência de complicação durante o transporte foram observados: distância do transporte superior a $100 \mathrm{~km}$, doença respiratória, presença de comorbidades e peso inferior a $10 \mathrm{~kg}$. Contudo, após a análise multivariada, nenhuma dessas variáveis se mantiveram como preditor independente para ocorrência de complicação durante o transporte. A presença de complicação durante o transporte foi associada com a maior taxa de mortalidade hospitalar mesmo após o ajuste para potenciais fatores de confusão.

A prevalência de complicações durante o transporte foi de $52 \%$ no presente estudo. Essa prevalência foi elevada comparando-se com dados da literatura, nos quais o transporte foi realizado por equipe especializada em transporte pediátrico, onde a prevalência de complicação foi em torno de 2 a 10\% (3). Em algumas localidades existem equipes especializadas no transporte pediátrico, como o Children's Acute Transport Service, que é um serviço de referência internacional na região de Londres, Inglaterra (4). No Brasil, o transporte dos pacientes dentro do sistema público de saúde é realizado pelo SAMU (2), o qual transporta uma grande proporção de adultos em comparação com um número menor de crianças, não havendo uma equipe especializada somente para o transporte pediátrico. Esse fato pode ser responsável pela prevalência elevada de complicação registrada durante o transporte dessas crianças gravemente doentes na região metropolitana de Ribeirão Preto. Apesar de não ser o objetivo deste estudo, os dados reforçam o conceito de que quando o transporte dessas crianças é realizado por equipe especializada há uma significativa redução na frequência de complicações $(3,5,6)$.
Neste estudo, observou-se que a complicação mais frequente foi relacionada aos dispositivos e a monitorização (42\%). Estudos recentes mostram que a otimização da monitorização no paciente pediátrico durante o transporte interhospitalar pode aumentar as intervenções e diminuir a incidência de disfunção de múltiplos órgãos, além de reduzir o tempo de internação hospitalar (7). A aferição contínua da pressão arterial foi associada à maior infusão de fluidos intravenosos e de administração precoce de drogas vasoativas quando comparada com a aferição intermitente (7). A monitorização inadequada dificulta o reconhecimento e a correção imediata de alterações oximétricas e hemodinâmicas, aumentando a frequência de complicações durante o transporte.

O peso inferior a $10 \mathrm{~kg}$ e a doença respiratória já foram identificados previamente como fatores preditivos no aumento de risco de morte e de permanência na unidade de tratamento intensivo pediátrica (8). Provavelmente, a doença respiratória aparece como preditor de complicação no transporte, pois esse tipo de doença está associada com a necessidade de intervenções em vias aéreas, que aumentam a probabilidade de ocorrência de complicações (9). Também é de fácil compreensão, que o peso baixo, nesse caso, inferior a $10 \mathrm{~kg}$, dificulte o manejo inicial emergencial da criança, aumentando o risco de complicações. Um estudo europeu comparou o transporte realizado por equipe especializada versus equipe não especializada em transporte pediátrico e mostrou que $88,5 \%$ dos pacientes com peso inferior a $15 \mathrm{~kg}$ não foram transportados com ventilador, pois esse não estava disponivel (10). A presença de comorbidade foi outro preditor de complicações, provavelmente, refletindo que crianças com problemas crônicos de saúde, como cardiopatias congênitas, doenças neurológicas e pulmonares, representam um grande desafio ao manejo clínico emergencial, principalmente, por médicos não especialistas em patologias pediátricas.

Em outro estudo que avaliou o transporte de pacientes adultos, a distância >60 milhas (96 km) foi considerada como fator prognóstico 
independente para a mortalidade (11). No entanto, dados recentes mostram que a duração do transporte, assim como a distância percorrida, não influenciam em desfechos clínicos, principalmente, se esse for realizado por equipes especializadas em transporte pediátrico que podem oferecer o mesmo nivel de cuidado recebido na unidade de tratamento intensivo $(8,12)$.

O dado mais relevante deste estudo foi mostrar que a presença de complicação durante o transporte foi preditor independentemente de mortalidade, mesmo após ajustes para potenciais fatores de confusão. Analisando-se as curvas de Kaplan-Meier para mortalidade intra-hospitalar, conclui-se que as curvas se separaram logo nos primeiros dias, provavelmente, refletindo o impacto rápido das complicações durante o transporte no desfecho de sobrevida.

No Reino Unido, a taxa de mortalidade dos pacientes transportados pelo Children's Acute Transport Service é de 6\% $(4,5)$ e, nos Estados Unidos, esse indice varia de 4 a 10\% $(13,14)$. Comparando-se pacientes internados na unidade de tratamento intensivo que foram transportados com os que não necessitaram de transporte, verificou-se uma taxa de mortalidade 3\% maior nos pacientes transportados, porém, após ajuste para o escore de gravidade, não houve diferença estatística na mortalidade (8). Esses estudos analisaram a mortalidade imediata após o transporte e, pelo nosso conhecimento, não foram encontrados estudos na literatura científica avaliando o impacto das complicações durante o transporte no desfecho de mortalidade no periodo subsequente de hospitalização.

Em relação a taxa de alta hospitalar, após os ajustes para potenciais fatores de confusão, não foi observada associação com a ocorrência de complicações durante o transporte. Provavelmente, outros fatores sejam mais importantes na determinação do tempo de hospitalização, como, por exemplo, a presença de comorbidades.

É importante ressaltar algumas limitações desta investigação. A primeira, está relacionada à representatividade da amostra, pois não houve uma sistematização para registrar a população de crianças que utilizaram o transporte hospitalar e que apresentavam os critérios de inclusão do estudo admitidos em nossa instituição durante o período do estudo. A inclusão de paciente dependia do contato do pesquisador com as unidades participantes, e com isso algumas falhas de inclusão podem ter ocorrido. A segunda, refere-se ao fato de os dados coletados serem oriundos de uma entrevista com o médico que admitiu o paciente no hospital e não com o médico responsável pelo transporte. Nesse aspecto, informações valiosas sobre intercorrências durante o transporte podem ter sido comprometidas, principalmente aquelas relacionadas a dispositivos e à monitorização. Por outro lado, a entrevista direta com esse médico poderia acarretar uma subnotificação de complicações. Devido ao desenho desse estudo, não se pode excluir a possibilidade de viés de recordação durante a entrevista com o médico. Dessa forma, é fundamental ressaltar a importância do registro detalhado das intercorrências observadas durante o transporte em formulário próprio pelos serviços que o realizam, contudo, essa documentação não é realizada adequadamente em nosso meio. A terceira limitação está relacionada à dificuldade em diferenciar se as complicações foram decorrentes do transporte propriamente dito ou do atendimento inicial prestado, principalmente, nos casos de transporte interhospitalar, contudo, pode-se afirmar que, caso houvesse complicações no local primário de atendimento, essas falhas poderiam ter sido solucionadas antes do transporte se uma equipe especializada em transporte pediátrico estivesse envolvida no processo. Por último, o escore PRISM2 não foi utilizado para ajuste estatístico da gravidade dos pacientes na análise multivariada e na regressão de Cox, pois já era esperado que pacientes com complicações durante o transporte apresentassem esse escore mais elevado na admissão e, deste modo, não representaria adequadamente a gravidade da doença que motivou o transporte.

Verificou-se elevada prevalência de 
complicações durante o transporte pré e interhospitalar de crianças gravemente doentes na região metropolitana de Ribeirão Preto. Além disso, a presença de complicações durante o transporte associou-se a um aumento na mortalidade intra-hospitalar. Julgamos necessário o desenvolvimento de um registro mais adequado das complicações observadas durante o transporte que permita um feedback imediato para a equipe com o objetivo de melhoria desse processo. Além disso, deve-se incentivar a implantação de serviços especializados em transporte pediátrico em grandes centros urbanos e o aprimoramento de recursos humanos e equipamentos em regiões menos populosas.

\section{Notas}

Parte deste trabalho é resultado de dissertação de mestrado profissional em Gestão de Organizações de Saúde de uma das autoras (TLG), da intitulada da Faculdade de Medicina de Ribeirão Preto da Universidade de São Paulo e foi apresentado ao 8th World Congress in Pediatric Intensive and Critical Care, Toronto, Canadá, 2016.

\section{Apoio financeiro}

Este estudo não recebeu apoio financeiro de fontes externas.

\section{Declaração de conflito de interesses}

Os autores declaram não haver conflitos de interesses relevantes ao conteúdo deste estudo.

\section{Contribuições dos autores}

Todos os autores fizeram contribuições substanciais para concepção, ou delineamento, ou aquisição, ou análise ou interpretação de dados; e redação do trabalho ou revisão crítica; e aprovação final da versão para publicação.

\section{Disponibilidade dos dados e responsabilidade pelos resultados}

Todos os autores declaram ter tido total acesso aos dados obtidos e assumem completa responsabilidade pela integridade destes resultados.

\section{Referências}

1. Henning R, McNamara V. Difficulties encountered in transport of the critically ill child. Pediatr Emerg Care. 1991;7(3):133-7. https://doi. org/10.1097/00006565-199106000-00001.

2. O'Dwyer G, Konder MT, Machado CV, Alves CP, Alves RP. The current scenario of emergency care policies in Brazil. BMC Health Serv Res. 2013;13:70. https://doi.org/10.1186/1472-6963-13-70.

3. Orr RA, Felmet KA, Han Y, McCloskey KA, Dragotta MA, Bills DM, et al. Pediatric specialized transport teams are associated with improved outcomes. Pediatrics. 2009;124(1):40-8. https://doi.org/10.1542/ peds.2008-0515.

4. Ramnarayan P, Polke E. The state of paediatric intensive care retrieval in Britain. Arch Dis Child. 2012;97(2):145-9. https://doi.org/10.1136/ adc. 2010.204503 .

5. Ramnarayan P, Thiru K, Parslow RC, Harrison DA, Draper ES, Rowan KM. Effect of specialist retrieval teams on outcomes in children admitted to paediatric intensive care units in England and Wales: a retrospective cohort study. Lancet. 2010;376(9742):698704. https://doi.org/10.1016/S0140-6736(10)61113-0.

6. Edge WE, Kanter RK, Weigle CG, Walsh RF. Reduction of morbidity in interhospital transport by specialized pediatric staff. Crit Care Med. 1994:22(7):1186-91. https://doi.org/10.1097/00003246-199407000-00023.

7. Stroud MH, Prodhan P, Moss M, Fiser R, Schexnayder S, Anand K. Enhanced monitoring improves pediatric transport outcomes: a randomized controlled trial. Pediatrics. 2011;127(1):42-8. https://doi. org/10.1542/peds.2010-1336.

8. Moynihan K, McSharry B, Reed P, Buckley D. Impact of Retrieval, Distance Traveled, and Referral Center on Outcomes in Unplanned Admissions to a National PICU. Pediatr Crit Care Med. 2016;17(2):e34-42. https://doi.org/10.1097/ PCC.0000000000000586.

9. Bigelow AM, Gothard MD, Schwartz HP, Bigham MT. Intubation in Pediatric/Neonatal Critical Care Transport: National Performance. Prehosp Emerg Care. 2015:19(3):351-7. https://doi.org/10.3109/10903 127.2014 .980481$.

10. Vos GD, Nieman FH, Meurs AM, Van Waardenburg DA, Ramsay G, Donckerwolcke RA. Problems in interhospital pediatric intensive care transport in The Netherlands: results from a survey of general pediatricians. Intensive Care Med. 2003;29(9):1555-9. https://doi.org/10.1007/s00134-003-1889-6.

11. Durairaj L, Will JG, Torner JC, Doebbeling BN Prognostic factors for mortality following interhospital transfers to the medical intensive care unit of a tertiary referral center. Crit Care Med. 2003:31(7):1981-6. https://doi.org/10.1097/01. CCM.0000069730.02769.16. 
12. McPherson ML, Graf JM. Speed isn't everything in pediatric medical transport. Pediatrics. 2009;124(1):3813. https://doi.org/10.1542/peds.2008-3596.

13. Odetola FO, Rosenberg AL, Davis MM, Clark SJ, Dechert RE, Shanley TP. Do outcomes vary according to the source of admission to the pediatric intensive care unit? Pediatr Crit Care Med. 2008;9(1):20-5. https://doi.org/10.1097/01.PCC.0000298642.11872.29.

14. Gregory CJ, Nasrollahzadeh F, Dharmar M, Parsapour K, Marcin JP. Comparison of critically ill and injured children transferred from referring hospitals versus in-house admissions. Pediatrics. 2008;121(4):eg06-11. https://doi.org/10.1542/peds.2007-2089.

\section{Endereço para correspondência}

Carlos Henrique Miranda

Centro Integrado de Emergências em Saúde, Unidade de Emergência - HC- FMRP-USP

Rua Bernardino de Campos, 1.000

$14015-130$

Ribeirão Preto, SP, Brasil 